\title{
Synergistic apoptosis-inducing effect of aspirin and isosorbide mononitrate on human colon cancer cells
}

\author{
XIAODONG WANG $^{1 *}$, YUWEN DIAO $^{1,2^{*}}$, YU LIU $^{1}$, NINGNING GAO $^{1}$, DONG GAO $^{1}$, \\ YANYAN WAN $^{1}$, JINGJING ZHONG ${ }^{1}$ and GUANGYI JIN ${ }^{1}$ \\ ${ }^{1}$ Shenzhen Engineering Laboratory of Synthetic Biology; \\ ${ }^{2}$ National-Regional Key Technology Engineering Laboratory for Medical Ultrasound, \\ School of Medicine, Shenzhen University, Shenzhen, Guangdong 518060, P.R. China
}

Received September 29, 2014; Accepted May 27, 2015

DOI: $10.3892 / \mathrm{mmr} .2015 .3963$

\begin{abstract}
Aspirin and isosorbide mononitrate (ISMN) are two commonly used drugs, which are clinically applied for the treatment of inflammatory and cardiovascular diseases, respectively. Recently, aspirin has attracted interest due to its potential application for the treatment of cancer, particularly colon cancer. NO-aspirin, an aspirin derivative containing a covalently bound NO-donating moiety, has been proven to be an effective anti-tumor agent with apoptosis-inducing ability. In the present study, ISMN was used as an NO donor and its synergic effect with aspirin was assessed in human colon cancer cells. In vitro, an MTT assay demonstrated that ISMN had a synergistic effect on the growth inhibitory effects of aspirin on HCT116 and SW620 colon cancer cells, while the growth of EA.hy926 normal endothelial cells was unaffected. This synergistic anti-tumor effect was further validated in vivo using nude mouse HCT116 cell xenograft model. Observation of nuclear morphology, Annexin V-fluorescein isothiocyanate/propidium iodide double staining and a caspase- 3 activity assay suggested that the combination of the two drugs induced apoptosis in HCT116 cells. Furthermore, the molecular mechanisms of the apoptotic effect of the drugs was assessed using an NO release assay, reverse transcription quantitative polymerase chain reaction analysis, western blot analysis and a luciferase reporter assay. It was certified that the increase in the amount of NO release, the decrease in the luciferase promoter activity and the expression of cyclin D1 and c-myc in HCT116 cells were affected by aspirin and ISMN in a synergistic manner. In conclusion, the present study
\end{abstract}

Correspondence to: Dr Guangyi Jin, Shenzhen Engineering Laboratory of Synthetic Biology, School of Medicine, Shenzhen University, 3688 Nanhai Avenue, Shenzhen, Guangdong 518060, P.R. China

E-mail: gyjin@szu.edu.cn

*Contributed equally

Key words: aspirin, isosorbide mononitrate, apoptosis, Wnt, NO was the first, to the best of our knowledge, to report on the synergistic apoptosis-inducing effects of aspirin and ISMN in human colon cancer cells, which were mediated via Wnt and NO signaling pathways. The results of the present study will facilitate the development of future therapeutic strategies.

\section{Introduction}

Aspirin is a well-known non-steroidal anti-inflammatory drug (NSAID), which has been used worldwide for hundreds of years. Studies have revealed that aspirin has numerous pharmacological effects, including cardioprotective and anti-tumor properties $(1,2)$. In gastroenterology, numerous emerging studies have suggested a potential application of aspirin in the prevention of certain types of cancer (3). Results of recent meta-analyses have estimated that long-term aspirin use may reduce the incidence of esophageal and colorectal cancers by up to $30 \%$ (4). Furthermore, aspirin has demonstrated growth-inhibiting and apoptosis-inducting abilities in numerous colorectal cancer cell lines, including HCT116 and SW480 (5). However, aspirin has a major drawback with regard to its routine administration, namely the risk of upper gastrointestinal bleeding and peptic ulceration (6).

Isosorbide mononitrate (ISMN), an organic nitrate compound, is a commonly used drug for the treatment of cardiovascular diseases, including angina pectoris, acute myocardial infarction and congestive heart failure. ISMN can be metabolized in vivo to nitric oxide (NO), which is an intercellular messenger with a variety of biological effects in the cardiovascular, nervous, immune and other systems $(7,8)$. NO also has similar effects to those of prostaglandins on gastric mucosa, which may reduce the gastric toxicity of aspirin (9). Furthermore, ISMN was shown to inhibit angiogenesis, tumor growth and metastasis in a chick model of the chorioallantoic membrane and a mouse model of Lewis Lung carcinoma (10).

Although aspirin and ISMN have been frequently used in the clinic, their effects on human colon cancer cells, particularly their synergistic anti-tumor effects, have remained elusive $(11,12)$. In the present study, the growth inhibitory effect of the combination of aspirin and ISMN was assessed using an MTT assay in the colon cancer cell lines HCT116 and SW620 and the umbilical vein cell line EA.hy926. 
The effects of the two drugs on cell apoptosis were further demonstrated through nuclear morphology observation, Annexin V-fluorescein isothiocyanate (FITC)/propidium iodide (PI) double staining, caspase-3 activity assay and detection of poly(adenosine triphosphate ribose) polymerase (PARP) cleavage. Additional experiments for the elucidation of the underlying mechanism of the apoptosis-inducing effects of the two drugs were performed by NO activation assay, luciferase reporter assay and analysis of Wnt pathway-associated signaling molecules. Finally, the synergistic anti-tumor effects were validated in a nude mouse HCT116 cell xenograft model in vivo.

\section{Materials and methods}

Compounds. Aspirin (no. A2093) and ISMN (no. I0775010) were standard substances purchased from Sigma-Aldrich (St Louis, MO, USA). They were pre-dissolved in dimethylsulfoxide (DMSO; Sigma-Aldrich) at a concentration of $2 \mathrm{~mol} / \mathrm{l}$.

Cell culture and drug treatments. HCT116 (no. CCL-247) and SW620 (no. CCL-227) human colon cancer cells and EA.hy926 (no. CRL-2922) human umbilical vein cells (American Type Cell Collection, Manassas, VA, USA) were cultured on Dulbecco's modified Eagle's medium (Gibco Life Technologies, Carlsbad, CA, USA) supplemented with $10 \%$ fetal bovine serum (Gibco Life Technologies), $100 \mathrm{U} / \mathrm{ml}$ penicillin and $100 \mu \mathrm{g} / \mathrm{ml}$ streptomycin (Gibco Life Technologies) in $25-\mathrm{cm}^{2}$ culture flasks at $37^{\circ} \mathrm{C}$ in a humidified atmosphere containing $5 \% \mathrm{CO}_{2}$. All cells to be tested in assays had a passage number of 3-6. For the drug treatment experiments, cells were harvested from the culture during the exponential growth phase, and then seeded into multi-well culture plates at $5 \times 10^{4}-1 \times 10^{5}$ cells $/ \mathrm{ml}$ in fresh medium. After attachment of the cells overnight, they were treated with the compounds at the indicated concentrations for $48 \mathrm{~h}$.

MTT assay for cell viability. At the end of the drug treatment period $(0.125,0.25,0.5$ or $1 \mathrm{mM}$ aspirin with 0,2 or $4 \mathrm{mM}$ ISMN) for $48 \mathrm{~h}, 10 \mu 15 \mathrm{mg} / \mathrm{ml}$ MTT solution (Sigma-Aldrich) in phosphate-buffered saline (PBS) (PBS without MTT as the blank) was added to each well of the culture plate (containing $100 \mu \mathrm{l}$ medium). After $4 \mathrm{~h}$ of incubation, the formazan crystals that formed in the wells were solubilized with $100 \mu \mathrm{l}$ DMSO for optical density reading at $570 \mathrm{~nm}$ with a spectrophotometer (Epoch; BioTek, Winooski, VT, USA).

Detection of changes in nuclear morphology. At the end of the drug treatment period $(1 \mathrm{mM}$ aspirin with $4 \mathrm{mM}$ ISMN for $48 \mathrm{~h}$ ), the cells in each well were washed once with PBS and fixed with $4 \%$ formaldehyde in PBS at $4^{\circ} \mathrm{C}$ for $30 \mathrm{~min}$. The cells were then washed with PBS and stained with $1 \mu \mathrm{g} / \mathrm{ml}$ Hoechst 33258 (no. C1018; Beyotime Institute of Biotechnology, Shanghai, China) in PBS at $37^{\circ} \mathrm{C}$ for $15 \mathrm{~min}$ and then viewed under a fluorescent microscope (DMI3000 B; Leica, Wetzlar, Germany) to observe changes in nuclear morphology.

Caspase-3 activity assay. Caspase-3 activity of cells was determined using a caspase-3 colorimetric assay kit (no. C1116;
Beyotime Institute of Biotechnology) according to the manufacturer' instructions. Briefly, cells were re-suspended in the cell lysis buffer and protein concentrations of the supernatants were measured. The samples were then incubated at $37^{\circ} \mathrm{C}$ with reaction buffer and substrate for 1-2 h. The optical density of the assay solutions was measured at $405 \mathrm{~nm}$ with a spectrophotometer (Epoch; BioTek).

Annexin V-FITC/PI double staining. The apoptotic rate of the cells was assessed using an Annexin V-FITC apoptosis detection kit (no. C1063; Beyotime Institute of Biotechnology) according to the manufacturer's instructions. Briefly, cells were collected, washed twice with PBS and washed once with binding buffer. The cells were then stained with Annexin V-FITC/PI at room temperature in the dark for $15 \mathrm{~min}$, and the fluorescence was quantified by flow cytometry (FACSCalibur; BD Biosciences, Franklin Lakes, NJ, USA). Early apoptotic cells were identified by Annexin $\mathrm{V}^{+}$/ PI- staining.

$N O$ release assay. NO release of cells was determined in the culture medium using a nitrate/nitrite colorimetric assay kit (no. S0024; Beyotime Institute of Biotechnology) according to the manufacturer's instructions. Briefly, the cell culture medium was collected after drug treatment and incubated at $37^{\circ} \mathrm{C}$ with nicotinamide adenine dinucleotide phosphate, flavin adenine dinucleotide and nitrate reductase for $30 \mathrm{~min}$, then at $37^{\circ} \mathrm{C}$ with lactate dehydrogenase (LDH) buffer and $\mathrm{LDH}$ for $30 \mathrm{~min}$, and then at room temperature with Griess reagent I and II for $10 \mathrm{~min}$. The optical density of the assay solutions was measured at $540 \mathrm{~nm}$ with a spectrophotometer (Epoch; BioTek).

Luciferase reporter assay for transcription factor (TCF) binding. DNA transfections were performed on HCT116 cells in the logarithmic growth phase. First, $0.2 \mu \mathrm{g}$ TOPflash (no. 21-170) or FOPflash (no. 21-169) (Millipore, Billerica, MA, USA) and $0.02 \mu \mathrm{g}$ pRL-TK (no. E2241; Promega, Madison, WI, USA) were co-transfected into $1 \times 10^{5} \mathrm{HCT} 116$ cells in each well of the culture plate using Lipofectamine 2000 reagent (no. 11668-019; Invitrogen Life Technologies, Carlsbad, CA, USA). After $4 \mathrm{~h}$ of transfection, aspirin and ISMN were added ( $1 \mathrm{mM}$ aspirin with $4 \mathrm{mM}$ ISMN) and incubated for 48 h. The luciferase activity was then evaluated by the DualLuciferase Reporter Assay System (no. E1910; Promega) using a microplate reader (TriStar2 LB942; Berthold, Bad Wildbad, Germany).

Reverse transcription quantitative polymerase chain reaction (RT-qPCR). At the end of the drug treatment period $(1 \mathrm{mM}$ aspirin with $4 \mathrm{mM}$ ISMN for $48 \mathrm{~h}$ ), the cells in each well were lysed in TRIzol solution (Invitrogen Life Technologies). RNA was extracted with the RNAiso Plus kit (no. 9108; Takara, Otsu, Japan) according to the manufacturer's instructions and quantitated spectrophotometrically. Total RNA was used as a template for reverse transcription using the following protocol: Each 20- $\mu 1$ reaction contained 1X Moloney's murine leukemia virus (M-MLV) buffer, $125 \mu \mathrm{M}$ deoxynucleotide triphosphate, 100 pmol oligo dT18 primer, 100 units M-MLV reverse transcriptase, diethyl pyrocarbonate-treated water and $2 \mu \mathrm{g}$ total RNA. Briefly, RNA and oligo dT18 primer was 
Table I. Sequences of primers used for polymerase chain reaction analysis.

\begin{tabular}{|c|c|}
\hline Gene & Sequence \\
\hline c-myc & $\begin{array}{l}\text { Forward: TGAACACAGCGAATGTTTCC } \\
\text { Reverse: TTAGGAGCGCTCAGGTCTGT }\end{array}$ \\
\hline Cyclin D1 & $\begin{array}{l}\text { Forward: CAGGTTGGACAGTTCACAGG } \\
\text { Reverse: ACAGCTGGAGTTGGATGGAC }\end{array}$ \\
\hline GAPDH & $\begin{array}{l}\text { Forward: GATGACATCAAGAAGGTGGTG } \\
\text { Reverse: GCTGTAGCCAAATTCGTTGTC }\end{array}$ \\
\hline
\end{tabular}

incubated at $70^{\circ} \mathrm{C}$ for $10 \mathrm{~min}$ and then immediately placed on ice, after which the other components were added and incubated at $42^{\circ} \mathrm{C}$ for $1 \mathrm{~h}$ and then at $70^{\circ} \mathrm{C}$ for $15 \mathrm{~min}$. $\mathrm{qPCR}$ was performed using the CFX96 Real-Time PCR Detection System (Bio-Rad Laboratories, Inc., Hercules, CA, USA) by using SYBR Premix Ex Taq (no. RR420A; Takara) according to the manufacturer's instructions. Primer sequences for c-myc, cyclin D1 and GAPDH genes are shown in Table I. The 20- $\mu 1$ qPCR reaction mixtures contained $10 \mu 1$ SYBR Premix Ex Taq, $0.2 \mu \mathrm{M}$ forward and reverse primer each, $2 \mu \mathrm{l}$ cDNA and nuclease-free water. The program used for all genes consisted of a denaturing cycle of $30 \mathrm{sec}$ at $95^{\circ} \mathrm{C}, 40$ cycles of PCR $\left(95^{\circ} \mathrm{C}\right.$ for $5 \mathrm{sec}$ and $60^{\circ} \mathrm{C}$ for $\left.30 \mathrm{sec}\right)$, and a specific melting cycle (from $65^{\circ} \mathrm{C}$ to $95^{\circ} \mathrm{C}, 0.5^{\circ} \mathrm{C}$ increment for $5 \mathrm{sec}$ ) in order to confirm the specificity of the QPCR products. The product sizes were confirmed by $3 \%$ agarose gel electrophoresis using the DL500 DNA marker (Takara) and electrophoresis apparatus (PowerPac Basic Power; Bio-Rad Laboratories, Inc.). and ethidium bromide staining. Results were analyzed using the $2^{-\triangle \Delta \mathrm{CT}}$ method to compare the transcriptional levels of target genes normalized to GAPDH in each sample relative to the non-treated control.

Western blot analysis. At the end of the drug treatment period ( $1 \mathrm{mM}$ aspirin with $4 \mathrm{mM} \mathrm{ISMN} \mathrm{for} 48 \mathrm{~h}$ ), the cells in each well were disrupted using cell lysis buffer (no. P0013; Beyotime Institute of Biotechnology). The suspension was centrifuged at $12,000 \mathrm{xg}$ and $4^{\circ} \mathrm{C}$ for $5 \mathrm{~min}$, and the protein content of the supernatant was determined using a bicinchoninic acid assay (Beyotime Institute of Biotechnology). Equal quantities of protein samples were loaded onto $10 \%$ SDS-polyacrylamide gel (Bio-Rad Laboratories, Inc.) and then transferred onto a microporous polyvinylidene difluoride membrane (Bio-Rad Laboratories, Inc.). Western blotting was performed using a mouse anti-human c-myc monoclonal antibody (no. sc-40; $1: 500$ dilution), mouse anti-human cyclin D1 monoclonal antibody (no. sc-450; 1:500 dilution) (both Santa Cruz Biotechnology, Inc., Dallas, TX, USA), rabbit anti-human caspase-3 polyclonal antibody (no. 9662; 1:1,000 dilution), rabbit anti-human PARP polyclonal antibody (no. 9532; 1:1,000 dilution) or rabbit anti-human $\beta$-actin monoclonal antibody (no. 8457; 1:1,000 dilution) and horseradish peroxidase-conjugated anti-mouse or anti-rabbit secondary antibodies (nos. 7076 and 7074; 1:1,500 dilution) (all Cell Signaling Technology, Danvers, MA, USA). Protein bands were visualized using an enhanced chemiluminescence substrate (no. 32109; Pierce, Thermo Fisher
Scientific, Waltham, MA, USA) and a FluorChem Q Western Blot Imaging System (ProteinSimple, Santa Clara, CA, USA).

Tumor xenograft study. The protocol for the animal experiment performed in the present study was approved by the Laboratory Animal Ethics Committee of Shenzhen University (Shenzhen, China). A total of 20 female BALB/c nude mice (4-week-old; 18-20 g) were purchased from the Medical Laboratory Animal Center (Guangzhou, China). All mice were housed under constant laboratory conditions of a 12-h light/dark cycle and specific pathogen-free conditions, and fed with water and food ad libitum. After being acclimatized for 1 week, each nude mouse was inoculated subcutaneously into the right-hand side of the back with $2 \times 10^{6}$ HCT116 cells in order to establish xenograft tumors. After six days, all mice grew visible tumors and were randomly assigned to four groups of five mice each: Control ( $0.5 \%$ sodium carboxymethyl cellulose orally once a day), ISMN $(150 \mathrm{mg} / \mathrm{kg}$ orally once a day), aspirin $(50 \mathrm{mg} / \mathrm{kg}$ orally once a day), and aspirin + ISMN (two drugs used together orally once a day). Aspirin and ISMN were ground into powder in a mortar and pestle and suspended in $0.5 \%$ sodium carboxymethyl cellulose (Sigma-Aldrich). The mice were treated for 12 days, and body weights and tumor volumes were monitored every three days. Tumor dimensions were measured with vernier calipers, and tumor volumes were

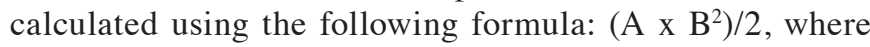
$\mathrm{A}$ is the larger and $\mathrm{B}$ is the smaller dimension of the tumor. Finally, all mice were anaesthetized using pentobarbital (Sigma-Aldrich), and the tumors were removed. The tumors were weighed and their images were captured.

Statistical analysis. Values are expressed as the mean \pm standard deviation of the indicated number of independently performed experiments. Student's t-test was used for the determination of statistical significance using SPSS 17.0 software (SPSS, Inc., Chicago, IL, USA). P $<0.05$ was considered to indicate a statistically significant difference between values.

\section{Results}

Aspirin and ISMN synergically inhibit the growth of colon cancer cells. After 48-h treatment of the HCT116 cells with aspirin (0-1 mM), the cell growth was dose-dependently inhibited relative to that of the untreated control. ISMN had a lower growth inhibitory effect than aspirin, as no obvious effect on HCT116 cell growth observed with $5 \mathrm{mM}$ ISMN and a growth inhibition rate of merely $10 \%$ was achieved with 10 mM ISMN (data not shown). In the present study, a non-cytotoxic concentration of ISMN ( 2 or $4 \mathrm{mM}$ ) was used to examine the synergic effect with aspirin on HCT116 cells. As shown in Fig. 1A, 1 mM aspirin had an inhibition rate of $51 \%$, while simultaneous treatment with 2 and $4 \mathrm{mM}$ ISMN increased the inhibition rate to 60 and 68\%, respectively. Similar results were achieved with lower concentrations of aspirin $(0.5,0.25$ and $0.125 \mathrm{mM})$. The $\mathrm{IC}_{50}$ (concentration causing 50\% growth inhibition of HCT116 cells) was $0.94 \pm 0.11 \mathrm{mM}$ for aspirin alone and $0.39 \pm 0.03 \mathrm{mM}$ in the presence of $4 \mathrm{mM}$ ISMN (Table II).

Similar experiments were performed on SW620 cells, another human colon cancer cell line, with similar results. Aspirin had a slightly lower cytotoxity on SW620 than on 
Table II. $\mathrm{IC}_{50}$ values $(\mathrm{mM})$ of aspirin in the absence or presence of ISMN on the human colon cancer cell lines HCT116 and SW620 and the human umbilican vein cell line EAhy.926.

\begin{tabular}{llll}
\hline ISMN added $(\mathrm{mM})$ & HCT116 & SW620 & EAhy926 \\
\hline 0 & $0.94 \pm 0.11$ & $1.28 \pm 0.20$ & $>>1$ \\
2 & $0.50 \pm 0.08^{\mathrm{a}}$ & $0.73 \pm 0.17^{\mathrm{a}}$ & $>>1$ \\
4 & $0.39 \pm 0.03^{\mathrm{b}}$ & $0.60 \pm 0.10^{\mathrm{b}}$ & $>>1$ \\
\hline
\end{tabular}

${ }^{\mathrm{a}} \mathrm{P}<0.05 ;{ }^{\mathrm{b}} \mathrm{P}<0.01$ vs no ISMN. ISMN, isosorbide mononitrate.
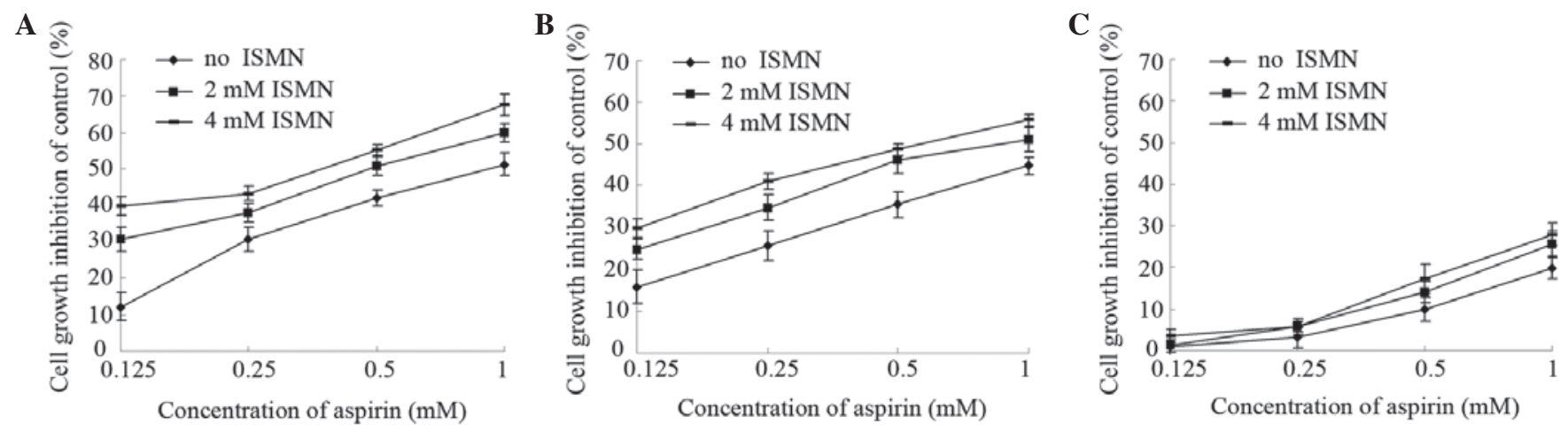

Figure 1. Growth inhibitory effect of aspirin on (A) HCT116, (B) SW620 and (C) EA.hy926 cells after 48-h treatment in the presence of ISMN (0, 2 or $4 \mathrm{mM})$. Values are expressed as the mean \pm standard deviation $(\mathrm{n}=3)$. ISMN, isosorbide mononitrate.

HCT116 cells (1 mM aspirin inhibited HCT116 growth by $51 \%$ and SW620 growth by 44\%). Furthermore, 4 mM ISMN had no obvious inhibitory effect on SW620 (data not shown). However, as shown in Fig. 1B, 2 and 4 mM ISMN enhanced the growth inhibition rate of $1 \mathrm{mM}$ aspirin on SW620 cells to 51 and $56 \%$, respectively. The $\mathrm{IC}_{50}$ on SW620 cells was $1.28 \pm 0.20 \mathrm{mM}$ for aspirin alone and $0.60 \pm 0.10 \mathrm{mM}$ in the presence of $4 \mathrm{mM}$ ISMN (Table II).

By contrast, markedly lower growth inhibitory effects were observed on non-cancerous EA.hy926 human umbilical vein cells treated with aspirin and/or ISMN. As shown in Fig. 1C, $1 \mathrm{mM}$ aspirin inhibited the growth of EA.hy926 cells by only $20 \%$. Compared with aspirin treatment alone, the inhibitory rate was not significantly altered upon co-treatment with non-cytotoxic concentrations of ISMN ( 2 or $4 \mathrm{mM}$ ). In summary, aspirin had a significantly greater growth inhibitory effect on human colon cancer cells compared with that on human umbilical vein cells. Furthermore, co-treatment with a non-cytotoxic concentration of ISMN inhibited the growth and viability of colon cancer cells in a synergistic manner with aspirin, while not affecting the growth of umbilical vein cells.

Aspirin and ISMN induce apoptosis in HCT116 cells. Changes of nuclear morphology, phosphatidylserine (PS) translocation and capase-3 activity of colon cancer cells were assessed to confirm cell apoptosis. After 48-h treatment, HCT116 cells were stained with Hoechst 33258 to observe chromatin condensation, which is a hallmark of apoptosis. None of the control cells showed specific chromatin condensation (Fig. 2A-a) and similarly, treatment with $4 \mathrm{mM}$ ISMN had no visible effects (Fig. 2A-b). However, in the presence of $1 \mathrm{mM}$ aspirin, hyperchromatic nuclei were observed in a percentage of the cells (Fig. 2A-c). A large increase in the number of cells with hyperchromatic nuclei cells was observed when $1 \mathrm{mM}$ aspirin and $4 \mathrm{mM}$ ISMN were used in combination (Fig. 2A-d).

Phosphatidylserine (PS) translocation from the inner to the outer side of the cell membrane is a characteristic event occurring in the early stage of apoptosis. Annexin V is a phospholipid-binding protein with high affinity to PS and is frequently used to prove the exposure of PS on the plasma membrane. To assess the apoptosis in the present study, flow cytometric analysis of Annexin V/PI double stained cells was performed. In the flow cytometry dot plots, Annexin $\mathrm{V}^{-} / \mathrm{PI}^{-}$[lower left (LL)] cells were designated as normal cells, Annexin $\mathrm{V}^{+} / \mathrm{PI}^{-}$[lower right (LR)] cells were designated as early apoptotic cells, Annexin $\mathrm{V}^{+} / \mathrm{PI}^{+}$[upper right (UR)] cells were designated as late apoptotic cells and Annexin $\mathrm{V}^{-} / \mathrm{PI}^{+}(\mathrm{UL})$ cells were designated as necrotic cells as shown in Fig. 2B and C. After 48-h treatment, few apoptotic cells were present in the control group and the $4 \mathrm{mM} \mathrm{ISMN}$ group (Fig. 2B-a and 2B-b). A significantly greater number of apoptotic cells were observed after $1 \mathrm{mM}$ aspirin treatment (Fig. 2B-c). Furthermore, when aspirin and ISMN were applied to HCT116 cells simultaneously, the population of early apoptotic cells further increased from $25.54 \pm 5.81$ to $48.06 \pm 2.56 \%$, compared to aspirin used alone (Fig. 2B-d). The percentages of late apoptotic cells (Annexin $\mathrm{V}^{+} / \mathrm{PI}^{+}$; UR) and necrotic cells (Annexin $\mathrm{V}^{-} / \mathrm{PI}^{+}$; UL) were not significantly changed by the drug treatments.

Caspase- 3 is the major executor caspase at the downstream of the apoptotic cascade, which is activated by other initiators and upstream caspases. The caspase-3 activity of HCT116 cells 
A
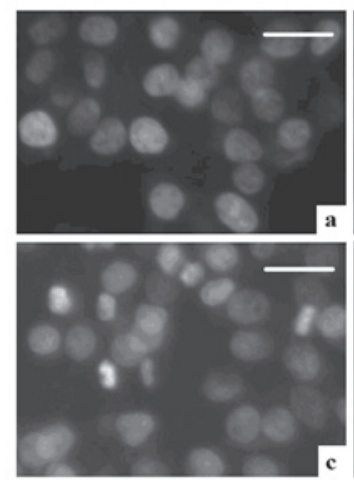
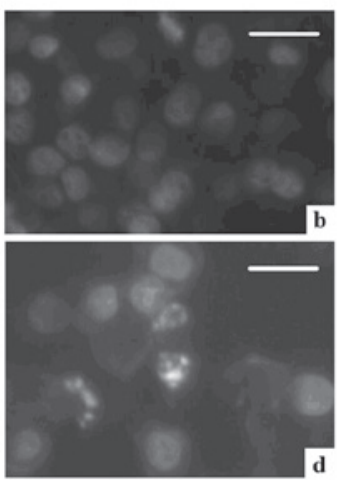

B
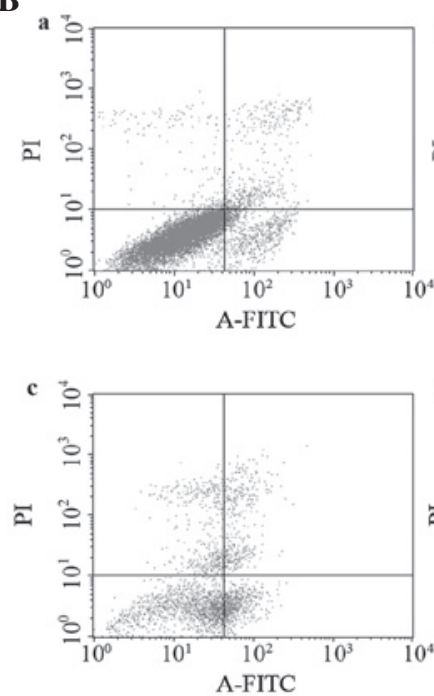
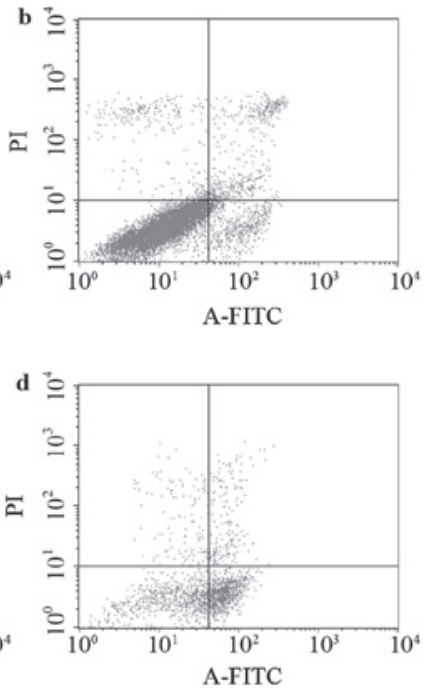

C

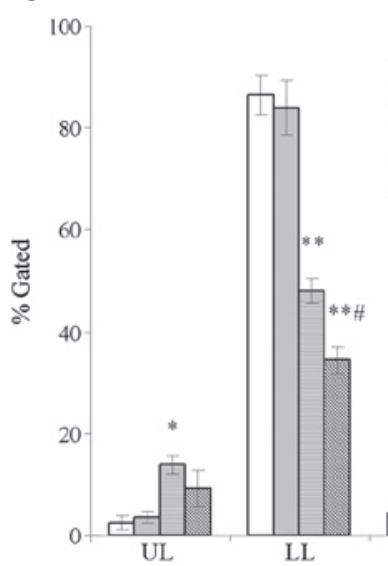

$\square$ Control

$\square$ ISMN

$\square$ Aspirin

목irin+ISMN

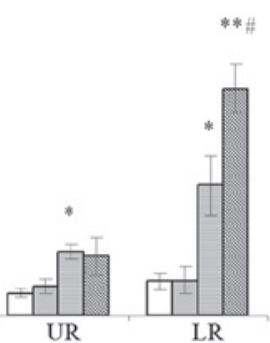

Figure 2. Apoptosis induction in HCT116 cells treated with $1 \mathrm{mM}$ aspirin and $4 \mathrm{mM}$ ISMN for $48 \mathrm{~h}$. (A) Nuclear morphology of HCT116 cells in (a) the control group and the groups treated with (b) ISMN, (c) aspirin and (d) aspirin + ISMN. Cells were stained with Hoechst 33258 and observed by fluorescence microscopy (scale bars, $20 \mu \mathrm{m}$ ). (B) Annexin V-FITC/PI analysis of HCT116 cells in (a) the control group and the groups treated with (b) ISMN, (c) aspirin and (d) aspirin + ISMN. (C) Quantified results of Annexin V-FITC/PI staining, where LL, LR, UL and UR quadrants represent normal, early apoptotic, necrotic and late apoptotic populations, respectively. Values are expressed as the mean \pm standard deviation $(\mathrm{n}=3)$. ${ }^{*} \mathrm{P}<0.05 ;{ }^{* *} \mathrm{P}<0.01$ vs. control group; ${ }^{\#} \mathrm{P}<0.05$ vs. aspirin group. FITC, fluorescein isothiocyanate; PI, propidium iodide; UL, upper left; UR, upper right; LL, lower left; LR, lower right; ISMN, isosorbide mononitrate.
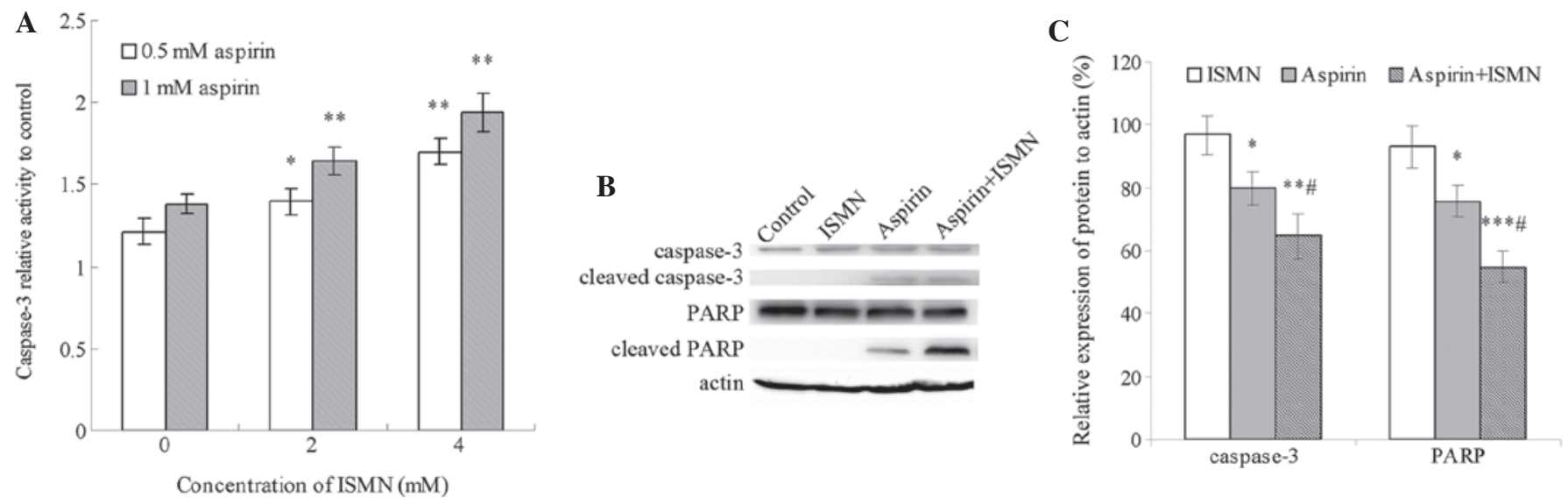

Figure 3. Caspase-3 activation in HCT116 cells induced by aspirin and ISMN after $48 \mathrm{~h}$ treatment. (A) Changes in caspase-3 activity following incubation with various concentrations of aspirin and ISMN. Values are expressed as the mean \pm standard deviation $(n=3) .{ }^{*} \mathrm{P}<0.05 ;{ }^{* * *} \mathrm{P}<0.01$ vs. aspirin group. (B) Effect of $1 \mathrm{mM}$ aspirin and $4 \mathrm{mM}$ ISMN on the cleavage of caspase-3 and PARP evaluated by western blot. (C) Quantification of caspase-3 and PARP expression by densitometric analysis. Values are expressed as the mean \pm standard deviation $(n=3) .{ }^{*} \mathrm{P}<0.05 ;{ }^{* * *} \mathrm{P}<0.01 ;{ }^{* * * *} \mathrm{P}<0.001$ vs. control group; ${ }^{*} \mathrm{P}<0.05$ vs. aspirin group. ISMN, isosorbide mononitrate; PARP, poly(adenosine triphosphate ribose) polymerase.

treated with the drugs for $48 \mathrm{~h}$ relative to that of the control group (no drug treatment) is shown in Fig. 3A. Treatment with 0.5 and $1 \mathrm{mM}$ aspirin caused a 1.2- and 1.4-fold increase in relative caspase-3 activity, respectively. By contrast, even 


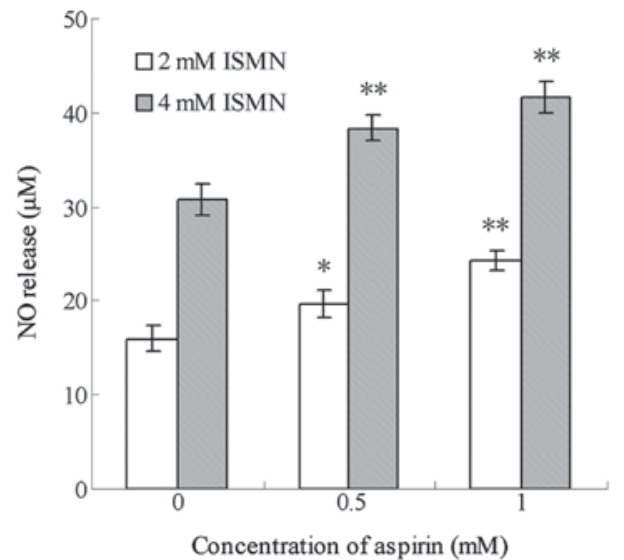

Figure 4. NO release was measured in the culture medium of HCT116 cells after 48-h treatment with various concentrations of aspirin and ISMN. Values are expressed as the mean \pm standard deviation $(n=3)$. ${ }^{*} \mathrm{P}<0.05 ;{ }^{* *} \mathrm{P}<0.01$ vs. ISMN group. ISMN, isosorbide mononitrate.

$10 \mathrm{mM}$ ISMN had no obvious impact on caspase-3 activity (data not shown). Of note, caspase-3 was markedly activated when ISMN was used in combination with aspirin. Co-treatment with 2 and $4 \mathrm{mM}$ ISMN increased caspase-3 activity from 1.4-fold of the control group with $1 \mathrm{mM}$ aspirin alone to 1.65- and 1.9-fold, respectively. A similar synergistic effect was achieved with lower concentrations of aspirin and ISMN. Furthermore, caspase-3 activation and PARP cleavage were assessed by western blot analysis. PARP is a nuclear enzyme involved in the DNA repair process and is specifically cleaved by caspase- 3 in the process of apoptosis. As shown in Fig. 3B and C, the formation of cleaved caspase-3 and PARP was induced by $1 \mathrm{mM}$ aspirin alone, and was further increased by co-treatment with $4 \mathrm{mM}$ ISMN. In conclusion, co-treatment with a non-cytotoxic concentration of ISMN synergistically enhanced HCT116 cell apoptosis induced by aspirin treatment through the caspase pathway.

Aspirin enhances the NO release from ISMN in the media of HCT116 cells. ISMN is a drug regularly used in the clinic for the treatment of cardiovascular diseases, and which is able to release a certain amount of $\mathrm{NO}$ in vivo. In the present study, culture medium containing 0.5 and $1 \mathrm{mM}$ ISMN was shown to contain 15 and $31 \mu \mathrm{M}$ NO, while media treated with $1 \mathrm{mM}$ aspirin alone contained an insignificant amount of NO (data not shown). As shown in Fig. 4, after 48-h treatment, aspirin significantly enhanced the NO release from ISMN when the two drugs were used in combination. For example, $0.5 \mathrm{mM}$ aspirin increased the amount of NO released from $4 \mathrm{mM}$ ISMN from $30.72 \pm 1.74$ to $38.36 \pm 1.31 \mu \mathrm{M}$, while $1 \mathrm{mM}$ aspirin increased NO levels from $30.72 \pm 1.74$ to $41.64 \pm 1.60 \mu \mathrm{M}$. Similar trends were observed with $2 \mathrm{mM}$ ISMN.

ISMN enhances the inhibitory effects of aspirin on the $\beta$-catenin/TCF signaling pathway in HCT116 cells. In colon cancer cells, such as HCT116, Wnt signaling is frequently activated as a result of the cellular accumulation of $\beta$-catenin. The TOPflash/FOPflash reporter gene assay was performed to investigate the effect of aspirin and ISMN on the transcriptional activity of $\beta$-catenin/TCF in HCT116 cells. TOPflash
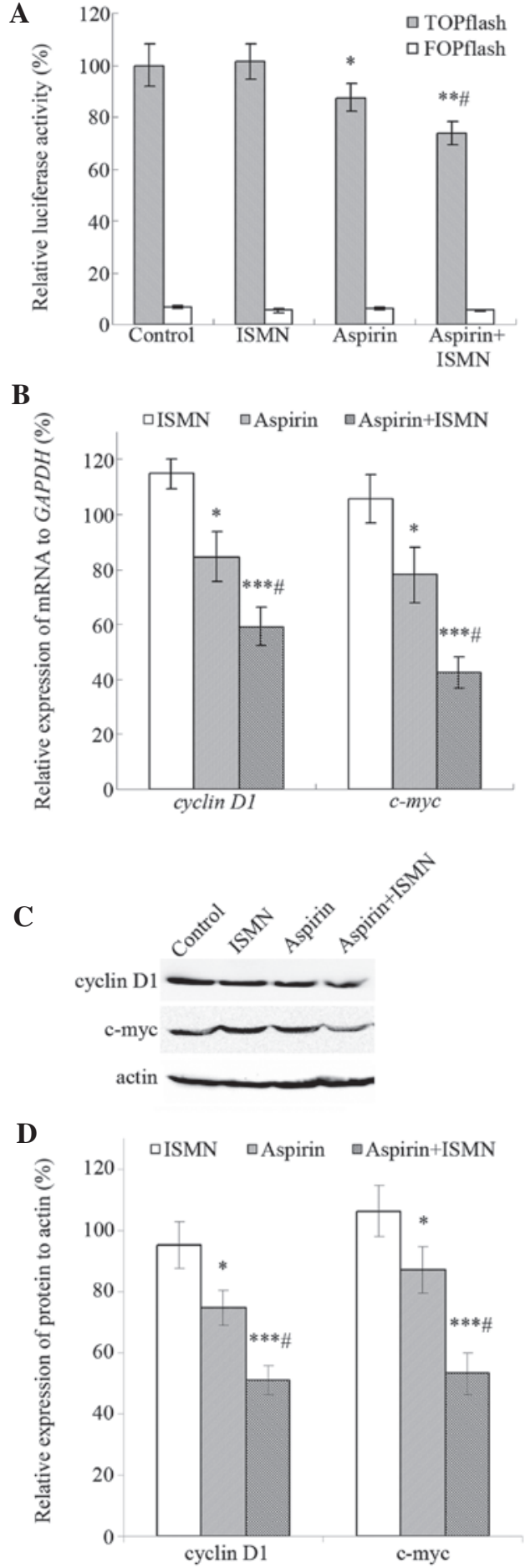

Figure 5. Inhibitory effects of aspirin $(1 \mathrm{mM})$ and ISMN $(4 \mathrm{mM})$ on the $\beta$-catenin/TCF signaling pathway in HCT116 cells following $48 \mathrm{~h}$. (A) Relative luciferase activity in HCT116 cells subjected to the TOPflash/FOPflash reporter gene assay. (B) Effect of aspirin and ISMN on the transcription levels of cyclin D1 and c-myc according to reverse transcription quantitative polymerase chain reaction analysis. (C) Effect of aspirin and ISMN on the translation of cyclin D1 and c-myc evaluated by western blot analysis. (D) Quantified protein levels of cyclin D1 and c-myc expression obtained by densitometric analysis of the western blots. Values are expressed as the mean \pm standard deviation $(n=3)$; ${ }^{*} \mathrm{P}<0.05 ;{ }^{* *} \mathrm{P}<0.01 ;{ }^{* * * *} \mathrm{P}<0.001$ vs. control group; ${ }^{\text {"P }} \mathrm{P}<0.05$ vs. aspirin group. ISMN, isosorbide mononitrate; TCF, transcription factor.

(TCF reporter plasmid) contains two sets of three copies of the TCF binding site (wild-type) upstream of the thymidine kinase minimal promoter, and FOPflash contains mutated 
A

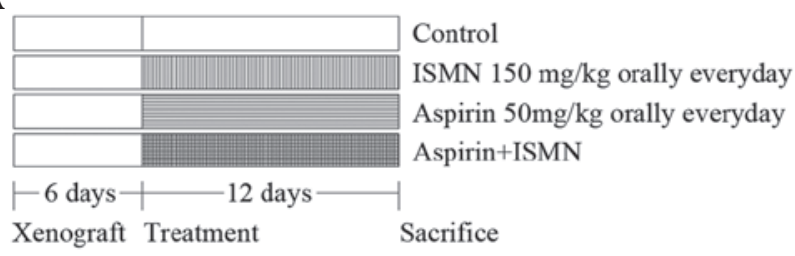

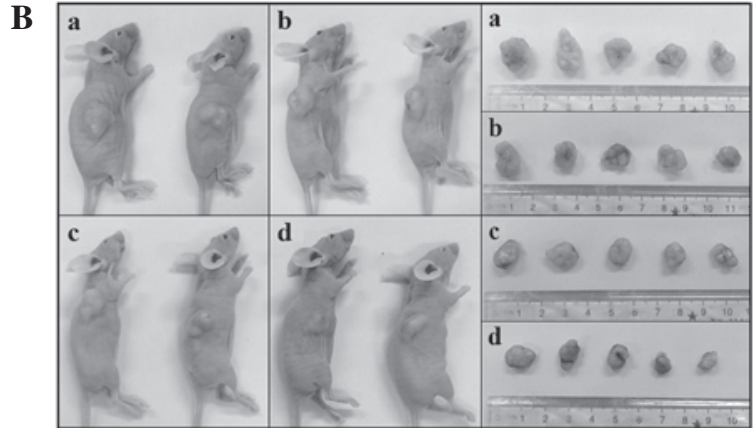

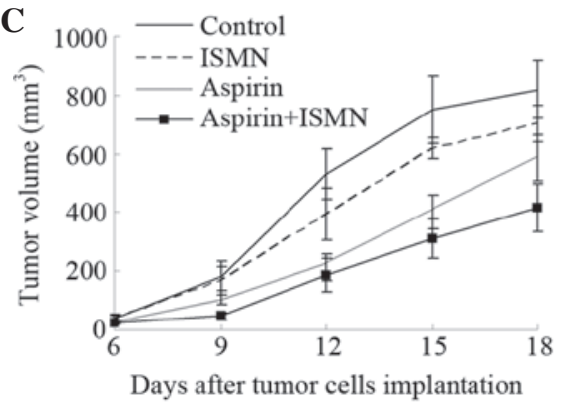

D 0.6

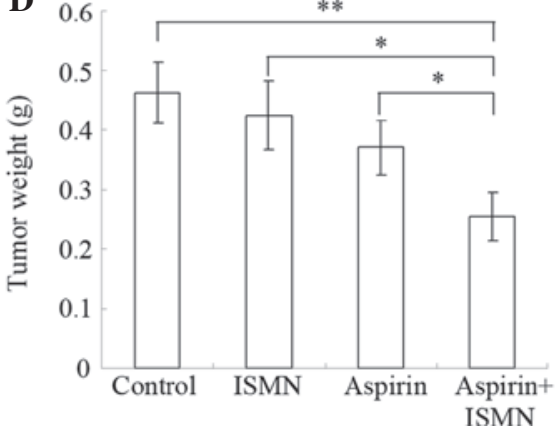

$\mathbf{E}$

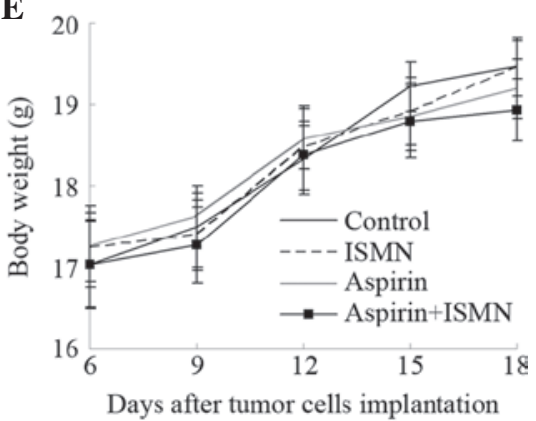

Figure 6. Effect of aspirin and ISMN on tumor growth in human colon cancer xenograft mice. (A) Scheme illustrating the experimental procedure of the tumor xenograft study. HCT116 cells were implanted subcutaneously into the flank of BALB/c nude mice on day 0. Drug treatment was performed on days 6-18. (B) Representative images of xenograft mice treated with (a) phosphate-buffered saline as a vehicle control, (b) ISMN, (c) aspirin and (d) aspirin + ISMN. (C) Growth curves depicting tumor volumes. Tumor dimensions were measured every three days and tumor volumes were calculated. (D) Tumor weights were determined at the end of the study. (E) Body weight of xenograft mice measured every three days. Values are expressed as the mean \pm standard deviation ( $\mathrm{n}=5$ ). ${ }^{*} \mathrm{P}<0.05,{ }^{* *} \mathrm{P}<0.01$. ISMN, isosorbide mononitrate.

TCF binding sites as a negative control. As shown in Fig. 5A, cells transfected with TOPflash showed significantly higher luciferase activity than those transfected with FOPflash, and TOPflash was markedly downregulated by the drugs rather than FOPflash. The luciferase activity of TOPflash decreased to $88 \%$ after treatment with $1 \mathrm{mM}$ aspirin, while $4 \mathrm{mM}$ ISMN had no impact on TOPflash. In addition, luciferase activity was further reduced to $73 \%$ when aspirin and ISMN were used in combination.

Cyclin D1 and c-myc are well-established target genes of the $\beta$-catenin-dependent pathway and have an important role in tumor cell growth and proliferation. RT-qPCR and western blot analyses were used to assess the transcription and translation of these $\beta$-catenin/TCF target genes. After 48-h treatment, $4 \mathrm{mM}$ ISMN did not alter the expression of the respective genes, while $1 \mathrm{mM}$ aspirin suppressed the mRNA and protein expression of cyclin D1 and c-myc. Furthermore, co-treatment of ISMN in addition to aspirin further reduced the transcription and translation of cyclin D1 and c-myc (Fig. 5B-D).

Tumor growth inhibition of aspirin and ISMN in xenograft mice. The synergistic inhibitory effect of aspirin and ISMN on colon cancer growth was further examined in vivo in a nude mouse xenograft model inoculated with HCT116 cells (Fig. 6). $50 \mathrm{mg} / \mathrm{kg}$ aspirin and/or $150 \mathrm{mg} / \mathrm{kg}$ ISMN were administered orally to the mice once a day starting from days 6-18 following tumor cell inoculation, as illustrated in Fig. 6A. Combined treatment of aspirin and ISMN resulted in smaller tumors compared with those in the single treatment or control groups (Fig. 6B-D). Even though aspirin or ISMN alone inhibited tumor volumes in the xenograft mice, combined treatment had a significantly more potent inhibitory effect (Fig. 6B-D). As shown in Fig. 6D, although treatment with ISMN or aspirin alone decreased the tumor weights, the differences compared with the control group were not significant. However, the average tumor weight in the combination treatment group (255 mg) was significantly lower than that in the control group (463 mg; P<0.01), ISMN group (424 mg; $\mathrm{P}<0.05$ ) or aspirin group $(370 \mathrm{mg} ; \mathrm{P}<0.05)$. Throughout the experiment, no significant difference of body weight was observed between the control and any treated group, indicating that no observable side effects emerged from the drug treatment (Fig. 6E).

\section{Discussion}

NO, first described as an endothelium-derived relaxation factor, is a free radical acting as a gaseous messenger that affects various biological functions (13). At low concentrations, NO functions as a signal transducer in numerous physiological processes, including as blood flow regulation, smooth muscle relaxation and iron homeostasis. At high concentrations, it also has a cytotoxic defensive role against pathogens and tumors (14). On the other hand, numerous studies indicated that chronic NO-level elevation may lead to certain pathological conditions in humans, including inflammatory bowel disease and cancer $(15,16)$. Although the association between NO and the biology of cancer has remained to be fully elucidated, NO-based anti-cancer drugs are being studied in vitro and in vivo to develop novel therapies (17). One of the successful examples are NO-NSAIDs, which consists of traditional 
NSAIDs covalently bound to an NO-donating moiety. It has been demonstrated that NO-NSAIDs not only possess a lower gastric toxicity, but are also more potent against colon carcinoma and other tumor types than parent NSAIDs (18). Among them, NO-aspirin is the most effective known candidate as indicated by studies which reported its efficacy in an animal model of cancer prevention (19) and its apoptosis-inducing ability in leukemia cells (20). In the present study, ISMN was used as an NO donor and its ability to promote the cytotoxicity of aspirin on tumor cells was examined.

The results of the present study showed that aspirin and ISMN had a synergistic inhibitory effect on HCT116 and SW620 cells, but not on EA.hy926 cells, indicating the specificity of the two drugs for human colon cancer cells. Aspirin alone exerted a certain cytotoxicity to HCT116 and SW620 cells, and was able to induce apoptosis in HCT116 cells after 48-h treatment, which is consistent with the findings of previous studies (5). ISMN alone only had a minor effect on cell growth and proliferation. However, when $4 \mathrm{mM}$ ISMN was used in combination with aspirin the $\mathrm{IC}_{50}$ value of aspirin on colon cancer cell lines dropped by $50 \%$ (from 0.94 to $0.39 \mathrm{mM}$ for HCT116, from 1.28 to $0.60 \mathrm{mM}$ for SW620). In addition, ISMN enhanced the apoptosis-inducing effect of aspirin on HCT116 cells, as evidenced by changes in nuclear morphology, PS translocation, caspase-3 activation and PARP cleavage. In the presence of $4 \mathrm{mM}$ ISMN, aspirin treatment resulted in a significantly larger amount of chromatin condensation, an elevated apoptotic rate and increased caspase- 3 activity. These results indicated that the induction of cell apoptosis, through a caspase-dependent pathway, is an underlying mechanism of the cytotoxicity of ISMN and aspirin to colon cancer cells.

Next, the present study performed an NO release assay in an attempt to provide an explanation for the molecular mechanism of the anti-tumor effects of ISMN. In the cell culture media, treatment with ISMN alone caused a release of NO in a concentration-dependent manner, while aspirin alone had no impact on NO levels in the media. However, in the presence of ISMN, a correlation was found between the concentration of aspirin and the amount of NO in the culture medium. Although ISMN is an NO donor, the NO increase from two drugs compared with ISMN alone was likely to originate from the cells through signaling pathway activation. When the two drugs were used at the same time, the amount of NO release was not completely in parallel with the cytotoxicity and apoptosis-inducing ability, indicating that other mechanisms may exist besides the activation of the NO pathway.

Furthermore, the effect of the drugs on the Wnt signaling pathway was thoroughly examined using a luciferase reporter assay, RT-qPCR and western blot analysis. In recent years, aberrant regulation of the Wnt pathway has been a prevalent theme in cancer biology. The $\mathrm{Wnt} / \beta$-catenin pathway, also called the canonical pathway, is well understood and controls cellular processes, including the cell cycle, apoptosis and differentiation (21). When Wnt signaling is activated, which leads to inactivation of the $\beta$-catenin destruction complex, $\beta$-catenin phosphorylation is reduced and stabilized. The stabilized $\beta$-catenin then translocates to the nucleus where it regulates downstream gene expression, cyclin D1 and c-myc as typical examples, by binding to lymphoid enhancer-binding factor/TCF factors (22). NSAIDs, including aspirin, have been shown to repress the function of $\beta$-catenin as a Wnt inhibitor to overcome malignant cells (23). NO-NSAIDs have also been reported to have marked growth-inhibitory and apoptotic effects on human epidermoid carcinoma cells by targeting $\beta$-catenin (24). In a reporter assay performed in the present study, HCT116 cells were transfected with TOPflash (TCF reporter plasmid) containing two sets of three copies of the TCF binding site (wild-type) upstream of the thymidine kinase minimal promoter, and FOPflash, containing mutated TCF binding sites as a negative control (25). It was demonstrated that aspirin inhibited the promoter activity of TOPflash, but not that of FOPflash, as well as the transcription and translation of cyclin D1 and c-myc. Despite the fact that ISMN alone did not influence Wnt signaling, TOPflash activity and cyclin D1 or c-myc expression in HCT116 cells were further reduced in a synergistic manner when aspirin and ISMN were used in combination. These results implied that inhibition of the Wnt/ $\beta$-catenin pathway is an underlying mechanism of the synergistic apoptosis-inducing effect of the two drugs.

Finally, a nude mouse xenograft model inoculated with HCT116 cells was applied to examine the anti-tumor efficacy of aspirin and ISMN in vivo. Combined treatment with the two drugs led to a considerable reduction in the volumes and weights of xenografted colon tumors without any obvious side effects, compared to the effects of vehicle control or single drug treatment.

In conclusion, the present study demonstrated for the first time, to the best of our knowledge, that two commonly used drugs, aspirin and ISMN, synergistically exerted anti-tumor effects on human colon cancer cells, while being less cytotoxic to normal human umbilical vein cells. The underlying mechanisms of action included the induction of cell apoptosis, the activation of the NO pathway and the inhibition of the Wnt pathway. The potent anti-tumor effect of the drug combination was also validated in nude mouse xenograft model in vivo. Therefore, the combined treatment with aspirin and ISMN is potentially and potent method for treating human colon cancer. Compared with novel drugs, well-known approved drugs, such as aspirin and ISMN, have more clinical applications and levels of acceptance, due to their well-defined pharmacokinetic/dynamic properties and side effects. Continued efforts should be made to elucidate the signal transduction pathways in more detail. Further clinical investigations are required to validate the efficacy of the drug combination in humans.

\section{Acknowledgements}

The authors would like to thank the Natural Science Foundation of China (grant no. 81202396), the Science Foundation of Shenzhen (grant no. JCYJ20130326112757843) and the Science Foundation of Shenzhen University (grant no. 201146) for financial support of this study.

\section{References}

1. Hossain MA, Kim DH, Jang JY, Kang YJ, Yoon JH, Moon JO, Chung HY, Kim GY, Choi YH, Copple BL, et al: Aspirin induces apoptosis in vitro and inhibits tumor growth of human hepatocellular carcinoma cells in a nude mouse xenograft model. Int $\mathbf{J}$ Oncol 40: 1298-1304, 2012. 
2. Fuster V and Sweeny JM: Aspirin: A historical and contemporary therapeutic overview. Circulation 123: 768-778, 2011.

3. Dovizio M, Bruno A, Tacconelli S and Patrignani P: Mode of action of aspirin as a chemopreventive agent. Recent Results Cancer Res 191: 39-65, 2013.

4. Jankowska H, Hooper P and Jankowski JA: Aspirin chemoprevention of gastrointestinal cancer in the next decade. A review of the evidence. Pol Arch Med Wewn 120: 407-412, 2010.

5. Goel A, Chang DK, Ricciardiello L, Gasche C and Boland CR A novel mechanism for aspirin-mediated growth inhibition of human colon cancer cells. Clin Cancer Res 9: 383-390, 2003.

6. Cooper K, Squires H, Carroll C, Papaioannou D, Booth A Logan RF, Maguire C, Hind D and Tappenden P: Chemoprevention of colorectal cancer: Systematic review and economic evaluation. Health Technol Assess 14: 1-206, 2010.

7. Chen C, Hu JT, Tu YJ, Wu JC, Liang J, Gao LL, Wang ZG, Yang BF and Dong DL: Effects of isosorbide mononitrate on the restoration of injured artery in mice in vivo. Eur J Pharmacol 640: 150-156, 2010.

8. Kaufman R, Nunes I, Bolognese JA, Miller DL, Salotti D, McCarthy JM, Smith WB, Herman GA and Feig PU: Single-dose effects of isosorbide mononitrate alone or in combination with losartan on central blood pressure. J Am Soc Hypertens 4: 311-318, 2010.

9. Wallace JL and Del Soldato P: The therapeutic potential of NO-NSAIDs. Fundam Clin Pharmacol 17: 11-20, 2003.

10. Pipili-Synetos E, Papageorgiou A, Sakkoula E, Sotiropoulou G Fotsis T, Karakiulakis G and Maragoudakis ME: Inhibition of angiogenesis, tumour growth and metastasis by the NO-releasing vasodilators, isosorbide mononitrate and dinitrate. Br J Pharmacol 116: 1829-1834, 1995.

11. Usman MW, Luo F, Cheng H,Zhao JJ and Liu P: Chemopreventive effects of aspirin at a glance. Biochim Biophys Acta 1855: 254-263, 2015.

12. Tan Z, Shang X, Li L, Tian L, Ma Y, Peng Y and Gao L: Clinical study of isosorbide mononitrate treatment for angina pectoris in coronary heart disease. Exp Ther Med 5: 1133-1136, 2013.

13. Palmer RM, Ferrige AG and Moncada S: Nitric oxide release accounts for the biological activity of endothelium-derived relaxing factor. Nature 327: 524-526, 1987.
14. Ignarro L: Nitric oxide biology and pathobiology. 2nd edition. Academic Press, New York, 2009.

15. Shah V, Lyford G, Gores G and Farrugia G: Nitric oxide in gastrointestinal health and disease. Gastroenterology 126: 903-913, 2004.

16. Xu W, Liu LZ, Loizidou M, Ahmed M and Charles IG: The role of nitric oxide in cancer. Cell Res 12: 311-320, 2002.

17. Mocellin S, Bronte V and Nitti D: Nitric oxide, a double edged sword in cancer biology: Searching for therapeutic opportunities. Med Res Rev 27: 317-352, 2007.

18. Rigas B and Kashfi K: Nitric-oxide-donating NSAIDs as agents for cancer prevention. Trends Mol Med 10: 324-330, 2004.

19. Williams JL, Nath N, Chen J, Hundley TR, Gao J, Kopelovich L, Kashfi K and Rigas B: Growth inhibition of human colon cancer cells by nitric oxide (NO)-donating aspirin is associated with cyclooxygenase-2 induction and beta-catenin/T-cell factor signaling, nuclear factor-kappaB, and NO synthase 2 inhibition: Implications for chemoprevention. Cancer Res 63: 7613-7618, 2003.

20. Khan NI, Cisterne A, Baraz R, Bradstock KF and Bendall LJ: Para-NO-aspirin inhibits NF-kappaB and induces apoptosis in B-cell progenitor acute lymphoblastic leukemia. Exp Hematol 40: 207-215 e1, 2012

21. Yang Y, Yang JJ, Tao H and Jin WS: New perspectives on betacatenin control of cell fate and proliferation in colon cancer. Food Chem Toxicol 74: 14-19, 2014.

22. Yang Y: Wnt signaling in development and disease. Cell Biosci 2: 14, 2012.

23. Lu D, Cottam HB, Corr M and Carson DA: Repression of beta-catenin function in malignant cells by nonsteroidal antiinflammatory drugs. Proc Natl Acad Sci USA 102: 18567-18571, 2005.

24. Nath N, Liu X, Jacobs L and Kashfi K: Flurbiprofen benzyl nitrate (NBS-242) inhibits the growth of A-431 human epidermoid carcinoma cells and targets $\beta$-catenin. Drug Des Devel Ther 7: 389-396, 2013

25. Kang YJ, Park HJ, Chung HJ, Min HY, Park EJ, Lee MA, Shin Y and Lee SK: Wnt/ $\beta$-catenin signaling mediates the antitumor activity of magnolol in colorectal cancer cells. Mol Pharmacol 82: 168-177, 2012. 